\title{
Effects of 18-month Vildagliptin Treatment on Portal Vein Pressure and Hepatosteatosis
}

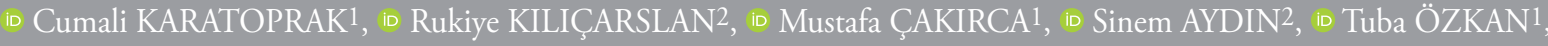 \\ ID Mehmet ZORLU1, iD Muharrem KISKAÇ1 \\ ${ }^{1}$ Bezmialem Vakif University Faculty of Medicine, Department of Internal Medicine, İstanbul, Turkey \\ 2Medipol University Faculty of Medicine, Department of Radiology, İstanbul, Turkey
}

\begin{abstract}
Objective: Patients with type 2 diabetes have an increased tendency to develop hepatosteatosis. The effects of drugs used to treat diabetes on the liver, regardless of the disease, are unknown. The aim of this study was to investigate the effects of vildagliptin, a dipeptidyl peptidase- 4 inhibitor, on the portal vein pressure and hepatosteatosis in patients with type 2 diabetes in the 18 months of follow-up.

Methods: Patients to whose treatment vildagliptin was added while they were on therapy with metformin and gliclazide for type 2 DM the vildagliptin group were included. As the control group, 49 patients with type $2 \mathrm{DM}$ treated with metformin and gliclazide were included. These patients were followed up for 18 months. These patients were followed for 18 months and their pre-treatment and post-treatment examinations were repeated. Portal vein diameter, portal vein flow and portal vein velocity were calculated to evaluate portal vein pressure with the same Doppler ultrasonography (US) by the same radiologist. In the same session, the liver steatosis stage of all patients was evaluated with US and recorded. The data before treatment and the data 18 months after treatment were compared.

Results: Nineteen patients completed the study in the study group, while 10 patients completed the study in the control group. A significant increase in portal vein flow velocity and vein diameter was found in the study group when portal vein parameters were compared before and after treatment $(\mathrm{p}=<0.001, \mathrm{p}=0.035$, respectively). There was no significant difference in portal vein flow volume. In the control group, no significant changes in flow velocity and flow volume were detected, although there was a significant increase in portal vein diameter $(\mathrm{p}=0.04, \mathrm{p}=0.07$, $\mathrm{p}=0.14$, respectively). There were no significant changes in vildagliptin group before and after treatment in terms of hepatosteatosis $(\mathrm{p}=0.41)$. There were no significant changes between control and study groups in terms of hepatosteatosis after 18 months of treatment.

Conclusion: As a result, we did not find any significant changes in the parameters of portal vein pressure with vildagliptin use. We think that vildagliptin has no effect on hepatosteatosis.
\end{abstract}

Keywords: Di-peptidyl peptidase 4 inhibitors, vildagliptin, portal vein pressure, hepatosteatosis, tip 2 diabetes mellitus

\section{Introduction}

Incretin-based therapies are agents that have been developed for the treatment of type 2 diabetes mellitus (DM). Improved glucoregulation has been observed following decreased break down or exogenous administration of an incretin hormone, glucagon-like peptide-1 (GLP-1). The advent of dipeptidyl peptidase-4 (DPP-4) inhibitors allows prevention of GLP-1 break down, thus increases its plasma concentration. Although these DPP-4 inhibitors act primarily on the pancreatic gland, they also exert effects on non-pancreatic organs $(1,2)$. The gastrointestinal system in particular, as well as the central nervous system, bone, fatty tissue and the cardiovascular system are the most affected organs $(3,4)$. This includes portal vein (PV) pressure and hepatic steatosis. In dog cell culture studies, there is an increased nitric

Address for Correspondence: Cumali Karatoprak, Bezmialem Vakif University Faculty of Medicine, Department of Internal Medicine, İstanbul, Turkey

E-mail: ckaratoprak@hotmail.com ORCID ID: orcid.org/0000-0001-7902-9284

Cite this article as: Cite this article as: Karatoprak C, Kiliçarslan R, Çakirca M, Aydin S, Ozkan T, Zorlu M, Kiskaç M Effects of 18-month Vildagliptin Treatment on Portal Vein Pressure and Hepatosteatosis. Bezmialem Science 2019;7(4):317-21. 
oxide (NO) release from endothelial cells associated with the increased levels of an incretin hormone, gastrointestinal peptide (GIP), and increased PV flow (5). NO is a short-living vasodilator with a significant role in the regulation of the vascular tonus (6). The effects of vildagliptin on PV pressure and hepatosteatosis were investigated previously (7). However, a long-term prospective study was not performed. In our study, we aimed to investigate the effects vildagliptin (DPP-4 inhibitor) on portal system pressure and hepatic steatosis by means of laboratory tests and portal system Doppler ultrasonography (US) in patients with type 2 DM following 18-month therapy.

\section{Method}

The study was designed as a prospective observational study. The study included patients to whose treatment vildagliptin $\left(2^{*} 50 \mathrm{mg}\right)$ was added while they were on therapy with metformin $\left(2^{*} 1000 \mathrm{mg}\right)$, gliclazide $\left(1^{*} 60 \mathrm{mg}\right)$ for type $2 \mathrm{DM}$ (the vildagliptin group). As the control group, patients treated with metformin $\left(2^{*} 1000 \mathrm{mg}\right)$ and gliclazide $\left(1^{*} 60 \mathrm{mg}\right)$ were included (the control group). Complete blood counts (CBCs) and biochemistry tests were performed in all subjects and their demographics were recorded. Each subject's PV diameter, PV flow volumeand PV flow velocity were measured with the same doppler US device and by the same radiologist to evaluate PV pressure. Hepatic steatosis was also graded with US during the same session. Both the vildagliptin group and the control group were followed up for a period of 18 months and subjects who were still on the same treatment at the end of 18 months and who did not meet the exclusion criteria underwent repeat PV doppler US. These subjects' CBCs and biochemistry tests were also repeated and demographics were recorded again. Of these subjects, vildagliptin users were evaluated in the pre-treatment and post- treatment periods. The subjects in the control group who did not receive vildagliptin were evaluated in the pretreatment and post- treatment periods. The obtained results were compared at the end of the study.

The study included patients who were at least 18 years of age, treated with gliclazide and metformin only or with vildagliptin added to gliclazide and metformin therapy. Patients with chronic liver disease which may increase portal pressure, congestive heart failure, chronic renal failure, chronic obstructive pulmonary disease, vasculitis and active infectious disease were excluded. In addition, patients receiving agents that are known to act on portal pressure including beta-blockers and isosorbide mononitrate were also excluded. Further exclusion criteria included patients treated with agents that are known to induce NO release including angiotensin converting enzyme blockers, angiotensin receptor blockers, calcium channel blockers. The study was initiated after it was approved by both the Ministry of Health and Ethics Board of Bezmialem Vakif University.

Doppler US method: The patients were instructed to fast after midnight and the analysis was performed between 09-12 a.m. Logiq 9 (GE, Milwaukee, USA) US and $3.5 \mathrm{mHz}$ convex probe were used for the analysis when the patient was lying on left lateral decubitus position. First, all liver segments were examined on gray scale and presence and degree of hepatosteatosis were recorded. $\mathrm{PV}$ measurements were performed on portal confluence. Doppler degree was maintained at $30^{\circ}-60^{\circ}$. Appropriate Doppler gain and filter adjustments were made. PV spectrum was recorded for at least 5 seconds mid-inspiration and measurements were made over this wave pattern. PV diameter, flow pattern, flow velocity and flow volume were assessed. These parameters were measured three times for each patient and the mean of measurements was taken.

Venous blood samples were obtained from the patients at least 1012 hours of fasting. Patients' blood samples were used to measure the following laboratory values: $\mathrm{Hb}_{\mathrm{lc}}$ (turbidimetric inhibition immunoassay; Roche), low-density lipoprotein cholesterol (LDL-C), triglyceride, hemogram, and alanine aminotransferase (ALT) and creatinine values (immunoassay chemiluminescent method; Beckman Coulter, Pasadena, CA, USA).

\section{Statistical Analysis}

SPSS (Statistical Package for Social Sciences) for Windows 15.0 software was used in statistical analyses.

During the assessment of study findings, Student's t-test and paired-samples t test were used to compare parameters with normal distribution. Mann-Whitney $U$ test and Wilcoxon were used to compare parameters without normal distribution. Chi-square and marginal homogeneity tests were employed to compare the proportional data. A two-sided $p$ value of $<0.05$ was considered significant.

\section{Results}

The study was initiated with 48 patients with type $2 \mathrm{DM}$ in the vildagliptin group. After 18 months of follow-up, and the study was completed with 19 participants, 5 of whom were male. On the other hand, the control group including the subjects who did not receive vildagliptin started the study with 49 patients with type $2 \mathrm{DM}$. This group completed the study with 10 subjects, 5 of whom were male. Demographics and laboratory values of subjects who were treated with vildagliptin did not differ significantly from those of the subjects in the control group. Pre- and post-treatment demographics and laboratory values of subjects in the vildagliptin group were mostly comparable. There was, however, a significant decrease in the creatinine level $(\mathrm{p}=0.02)$ and a significant increase in ALT level $(\mathrm{p}=0.02)($ Table 1). Pre- and post-treatment values in the control group did not differ significantly (Table 2 ).

PV flow velocity, PV flow volume and PV diameter were measured with doppler US in the control and the vildagliptin groups. Comparison of PV parameters in the vildagliptin group demonstrated significant increases in terms of PV flow velocity and PV diameter $(\mathrm{p}=<0.001, \mathrm{p}=0.035$, respectively). No significant difference was observed for PV flow volume. In the control group, no significant differences were seen for PV flow velocity or PV flow volume, although there was a significant increase in the PV diameter (Table 3). Comparison of 18-month therapy results between the vildagliptin group and the control 
group demonstrated no significant differences in the PV flow velocity, PV flow volume or diameter $(\mathrm{p}=0.66, \mathrm{p}=0.2, \mathrm{p}=0.67$, respectively).

Assessment of hepatosteatosis grades did not demonstrate any significant increases in terms of steatosis $(\mathrm{p}=0.157)$. There was no significant increase between the control and vildagliptin groups in terms of hepatosteatosis after 18 months of treatment (Table 4).

Vildagliptin and control groups' pre- and post-treatment white blood cell, hemoglobin, hemotocrit, mean corpuscular volume and platelet values did not differ significantly $(\mathrm{p}=0.98, \mathrm{p}=0.53$, $\mathrm{p}=0.35, \mathrm{p}=0.38, \mathrm{p}=0,29, \mathrm{p}=39, \mathrm{p}=0.94, \mathrm{p}=0.7, \mathrm{p}=0.27, \mathrm{p}=0.7$, respectively).

Power analysis calculation of the study based on PV flow velocity yielded a value of $98 \%$ in the vildagliptin group.

Table 1. The demographic and laboratory characteristics of the subjects by groups

\begin{tabular}{|c|c|c|c|}
\hline & $\begin{array}{l}\text { Pre vildagliptin } \\
(n=19)\end{array}$ & $\begin{array}{l}\text { Post vildagliptin } \\
(n=19)\end{array}$ & $p$ \\
\hline Age (years) & $52.16 \pm 7.18$ & & \\
\hline BMI $\left(\mathrm{Kg} / \mathrm{m}^{2}\right)$ & $33.3 \pm 5.5$ & $33.16 \pm 4.9$ & 0.72 \\
\hline Glucose (mg/dL) & $160.5 \pm 37$ & $155.5 \pm 33.8$ & 0.58 \\
\hline HbA1c (\%) & $7.16 \pm 1.08$ & $7.02 \pm 1$ & 0.8 \\
\hline Creatinine (mg/dL) & $0.75 \pm 0.14$ & $0.62 \pm 0.14$ & 0.02 \\
\hline $\operatorname{ALT}(\mathrm{U} / \mathrm{L})$ & $23.6 \pm 8.5$ & $31.9 \pm 13.9$ & 0.02 \\
\hline LDL-C (mg/dL) & $127.8 \pm 40.3$ & $121.8 \pm 61$ & 0.39 \\
\hline Triglyceride (mg/dL) & $153 \pm 60$ & $148 \pm 24.4$ & 0.98 \\
\hline
\end{tabular}

\section{Discussion}

DPP-4 inhibitors are demonstrated to reduce glucose and glucagon levels by increasing serum levels of GLP-1 and GIP hormones. These hormones are known to potentiate NO release by acting on the endothelia in several tissues (6). It is recognized that elevated levels of $\mathrm{NO}$ induce vasodilation in the PV, whereas decreased levels of glucagon are known to cause vasodilation in the splenic vein. In addition to these data, DPP-4 activity was found to be increased in patients with nonalcoholic hepatosteatosis, and hepatosteatosis grade was found to be higher with increasing levels of DPP-4 (8). Vildagliptin is a DPP-4 inhibitor which is recommended rarely in patients at risk of asymptomatic hepatitis, because its prescribing information advices hepatic function monitoring four times yearly. Based on these data, a decrease in PV pressure and increase in hepatic steatosis may be expected with short- and long-term vildagliptin use. In our study, we intended to investigate these potential effects of vildagliptin using non-invasive methods.

Table 2. The demographic and laboratory characteristics of the subjects by groups

\begin{tabular}{|c|c|c|c|}
\hline & $\begin{array}{l}\text { Pre control } \\
(n=10)\end{array}$ & $\begin{array}{l}\text { Post control } \\
(n=10)\end{array}$ & $p$ \\
\hline Age (years) & $55.4 \pm 9.2$ & & \\
\hline BMI $\left(\mathrm{Kg} / \mathrm{m}^{2}\right)$ & $30.8 \pm 6.3$ & $30.6 \pm 4.8$ & 0.8 \\
\hline Glucose (mg/dL) & $149.8 \pm 19.6$ & $156.8 \pm 35.3$ & 0.7 \\
\hline HbA1c (\%) & $6.75 \pm 0.5$ & $6.95 \pm 0.39$ & 0.58 \\
\hline Creatinine (mg/dL) & $0.83 \pm 0.15$ & $0.68 \pm 0.08$ & 0.07 \\
\hline ALT (U/L) & $16.5 \pm 5.3$ & $27.7 \pm 4.3$ & 0.09 \\
\hline LDL-C (mg/dL) & $124.3 \pm 42.2$ & $134.3 \pm 38.9$ & 0.08 \\
\hline Triglyceride (mg/dL) & $106.3 \pm 41$ & $130.3 \pm 116$ & 0.6 \\
\hline
\end{tabular}

Control group: Subjects on metformin and gliclazide, HbA1 c: Hemoglobin A1C, ALT: Alanine aminotransferase, LDL-C: Low-density lipoprotein cholesterol, BMI: Body mass index

\section{Table 3. Portal vein flow velocity, portal vein flow volume, and portal vein diameter by group}

\begin{tabular}{|c|c|c|c|c|c|c|}
\hline & $\begin{array}{l}\text { Pre vildagliptin } \\
(n=19)\end{array}$ & $\begin{array}{l}\text { Post vildagliptin } \\
(n=19)\end{array}$ & $P$ & $\begin{array}{l}\text { Pre control } \\
(n=10)\end{array}$ & $\begin{array}{l}\text { Post control } \\
(n=10)\end{array}$ & $P$ \\
\hline Portal vein flow velocity (L/min) & $7.8 \pm 1.2$ & $15.4 \pm 5.6$ & $<0.001$ & $8.4 \pm 2.3$ & $9.5 \pm 2.25$ & 0.07 \\
\hline Portal vein flow volume (cm/s) & $475 \pm 145$ & $428 \pm 182$ & 0.44 & $488 \pm 95$ & $599 \pm 139$ & 0.14 \\
\hline
\end{tabular}

Table 4. Comparison of steatosis grades with ultrasonography between groups in pre- and post-treatment periods

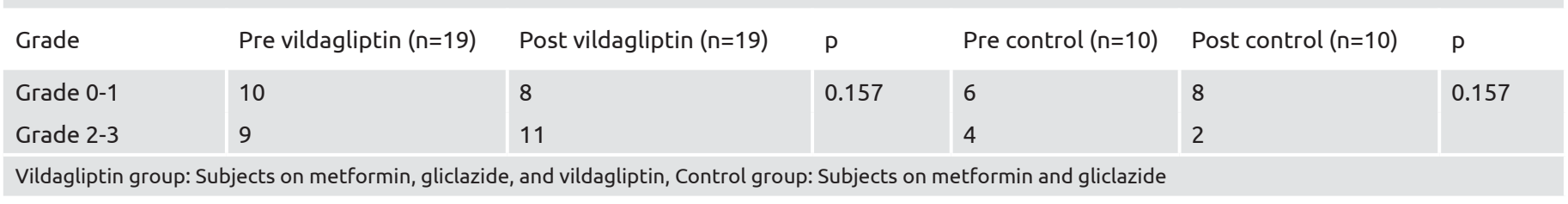


Significant increase in PV flow velocity and PV diameter was observed in subjects using vildagliptin in our study. These may be interpreted as a non-invasive indicator of reduced $\mathrm{PV}$ pressure (9). In vildagliptin users, increased PV diameter may be expected as a consequence of increased NO levels. There was no significant decrease in PV flow volume.

Reduced PV flow volume is recognized as an indicator of reduced PV pressure. Cell culture studies showed that exogenous GIP given to dog fetal cell culture increased NO release from PV (4). We could not identify any studies investigating the effect of DPP-4 inhibitors on NO in portal veins. There are, however, many studies on other tissues and organs. In one of these, obese rats were treated with saxagliptin and aortic and glomerular endothelial NO levels were measured. The authors showed significantly elevated endothelial $\mathrm{NO}$ levels in animals treated with saxagliptin (10). Another study demonstrated decreased levels of serum acetyl di-methyl arginine, which is recognized as an indirect indictor of $\mathrm{NO}$ elevations, in subjects receiving vildagliptin (11). These results may be supportive of the results obtained in our study. On the other hand, significantly increased $\mathrm{PV}$ diameter in the control group was not, in fact, an expected outcome. Absence of a significant difference in PV flow volume and PV flow velocity may indicate that PV pressure was not significantly affected. Vildagliptin seems to cause a reduction in PV pressure when the results obtained from the vildagliptin group and the control group were taken separately. However, when we compared the 18-month differences of the vildagliptin and control groups, we determined that PV parameters were all comparable between the groups. This contradiction may be explained as follows: although these parameters seemed to differ significantly when assessed in individual groups, the difference, in fact, was very smalland when the whole sample was evaluated, the difference was not significant. These results may suggest that the parameters which allowed indirect estimation of PV pressure were not altered to a significant extent with vildagliptin use.

Patients who completed the 18-month vildagliptin treatment did not differ significantly with regards to hepatosteatosis grade. In the control group, there were no significant differences in hepatosteatosis or serum ALT levels. A published review on the effects of DPP-4s on the liver included studies which reported that DPP-4 inhibitors corrected hepatic steatosis as well as those which described a close association with hepatic steatosis (12). A study investigating the effects of sitagliptin, a DPP-4 inhibitor, in patients with moderate hepatic impairment found that the drug was safe and did not cause clinical deterioration (13). Another study reported increased hepatosteatosis with increasing levels of DPP-4 (8). Our results did not indicate a significant increase in hepatic steatosis.

Another important outcome of the study was that there was a statistically significant decrease in serum creatinine levels in patients receiving vildagliptin versus no significant difference in the creatinine levels in the control group. A previous study found significantly reduced levels of urinary albumin creatinine in subjects who used vildagliptin for 8 weeks compared to controls (14). Another study demonstrated a significant decrease in creatinine levels in rats treated with vildagliptin (15). The results of these studies are consistent with the results of our study.

The limitation of our study was that we did not measure the hepatic venous pressure gradient with the invasive angiography, the golden standard of assessing PV pressure. Instead, we preferred portal doppler US which is a non-invasive method and is efficient in determining the severity of portal hypertension. PV flow velocity, diameter and flow volume measured with PV doppler US was reported to have a sensitivity and specificity of $80 \%$ in reflecting PV pressure $(16,17)$. Another limitation was that a low number of patients completed 18-month treatment in the study.

In conclusion, satisfactory evidence to suggest that vildagliptin use results in a significant decrease in PV pressure was not obtained. However, these results may suggest that vildagliptin does not increase PV pressure. This may be particularly important when selecting an agent for patients with PV pressure. It should also be kept in mind that increased NO release can be dangerous in patients who have esophageal varices or are at risk of having bleeding in esophageal varices. As the second outcome of the study, vildagliptin was not shown to have an effect on hepatosteatosis. This requires larger studies. Further studies are also needed to elucidate whether the obtained results are associated with vildagliptin or are class effects of DPP-4 inhibitors.

\section{Ethics}

Ethics Committee Approval: The study was initiated after it was approved by both the Ministry of Health and Ethics Board of Bezmialem Vakif University.

Informed Consent: Written consent was obtained from the students who agreed to participate in the study.

Peer-review: Externally peer-reviewed.

\section{Authorship Contributions}

Concept: C.K., Design: C.K., Data Collection or Processing: M.Z., M.K., Analysis or Interpretation: C.K., R.K., M.G., S.A., T.Ö., Writing: C.K.

Conflict of Interest: No conflict of interest was declared by the authors.

Financial Disclosure: The authors declared that this study received no financial support.

\section{Acknowledgments}

The authors declare that they have no competing interests.

\section{References}

1. Kim W, Egan JM. The role of incretins in glucose homeostasis and diabetes treatment. Pharmacol Rev 2008;60:470-512.

2. Deacon CF, Wamberg S, Bie P, Hughes TE, Holst JJ. Preservation of active incretin hormones by inhibition of dipeptidyl peptidase IV suppresses meal-induced incretin secretion in dogs. J Endocrinol 2002;172:355-62. 
3. Meier JJ, Goetze O, Anstipp J, Hagemann D, Holst JJ, Schmidt WE, et al. Gastric inhibitory polypeptide does not inhibit gastric emptying in humans. Am J Physiol Endocrinol Metab 2004;286:E621-5.

4. Baggio LL, Huang Q, Cao X, Drucker DJ. An albumin-exendin-4 conjugate engages central and peripheral circuits regulating murine energy and glucose homeostasis. Gastroenterology 2008;134:113747.

5. Ding $\mathrm{KH}$, Zhong $\mathrm{Q}$, $\mathrm{Xu}$ J, Isales CM. Glucose-dependent insulinotropic peptide: differential effects on hepatic artery vs. portal vein endothelial cells. Am J Physiol Endocrinol Metab 2004;286:E773-9.

6. He YL, Serra D, Wang Y, Campestrini J, Riviere GJ, Deacon CF, et al. Pharmacokinetics and pharmacodynamics of vildagliptin in patients with type 2 diabetes mellitus. Clin Pharmacokinet 2007;46:577-88.

7. Karatoprak C, Kiliçarslan R, Cakirca M, Aydin S, Ozkan T, Kocaman O, et al. Vildagliptin Treatment on the Portal Venous Pressure and Hepatosteatosis in Patients with Type 2 Diabetes Mellitus. Bezmialem Science 2018;6:1-5.

8. Balaban YH, Korkusuz P, Simsek H, Gokcan H, Gedikoglu G, Pinar A, et al. Dipeptidyl peptidase IV (DDP IV) in NASH patients. Ann Hepatol 2007;6:242-50.

9. Zironi G, Gaiani S, Fenyves D, Rigamonti A, Bolondi L, Barbara L. Value of measurement of mean portal flow velocity by Doppler flowmetry in the diagnosis of portal hypertension. J Hepatol 1992;16:298-303.

10. Mason RP, Jacob RF, Kubant R, Walter MF, Bellamine A, Jacoby A, et al. Effect of enhanced glycemic control with saxagliptin on endothelial nitric oxide release and CD40 levels in obese rats. J Atheroscler Thromb 2011;18:774-83.
11. Cakirca M, Karatoprak C, Zorlu M, Kiskac M, Kanat M, Cikrikcioglu $\mathrm{MA}$, et al. Effect of vildagliptin add-on treatment to metformin on plasma asymmetric dimethylarginine in type 2 diabetes mellitus patients. Drug Des Devel Ther 2014;8:239-43.

12. Itou M, Kawaguchi T, Taniguchi E, Sata M. Dipeptidyl peptidase-4: a key player in chronic liver disease. World J Gastroenterol 2013;19:2298-306.

13. Migoya EM, Stevens CH, Bergman AJ, Luo WL, Lasseter KC, Dilzer SC, et al. Effect of moderate hepatic insufficiency on the pharmacokinetics of sitagliptin. Can J Clin Pharmacol 2009;16:e16570.

14. Tani S, Nagao K, Hirayama A. Association between urinary albumin excretion and low-density lipoprotein heterogeneity following treatment of type 2 diabetes patients with the dipeptidyl peptidase- 4 inhibitor, vildagliptin: a pilot study. Am J Cardiovasc Drugs 2013;13:443-50.

15. Liu WJ, Xie SH, Liu YN, Kim W, Jin HY, Park SK, et al. Dipeptidyl peptidase IV inhibitor attenuates kidney injury in streptozotocininduced diabetic rats. J Pharmacol Exp Ther 2012;340:248-55.

16. Haag K, Rossle M, Ochs A, Huber M, Siegerstetter V, Olschewski M, et al. Correlation of duplex sonography findings and portal pressure in 375 patients with portal hypertension. AJR Am J Roentgenol 1999; 172:631-5.

17. Singal AK, Ahmad M, Soloway RD. Duplex Doppler ultrasound examination of the portal venous system: an emerging novel technique for the estimation of portal vein pressure. Dig Dis Sci 2010;55:1230-40. 